\title{
Ramas, familles, réseaux. Les supports sociaux de la diffusion de la santería cubaine (Cuba-Mexique)
}

Kali Argyriadis

\section{(2) OpenEdition}

1 Journals

Édition électronique

URL : https://journals.openedition.org/jsa/3022

DOI : 10.4000/jsa.3022

ISSN : $1957-7842$

Éditeur

Société des américanistes

Édition imprimée

Date de publication : 5 décembre 2005

Pagination : 153-183

ISSN : 0037-9174

\section{Référence électronique}

Kali Argyriadis, «Ramas, familles, réseaux. Les supports sociaux de la diffusion de la santería cubaine (Cuba-Mexique) », Journal de la Société des américanistes [En ligne], 91-2 | 2005, mis en ligne le 10 juin 2010, consulté le 05 septembre 2022. URL : http://journals.openedition.org/jsa/3022 ; DOI : https://doi.org/10.4000/jsa.3022 


\title{
RAMAS, FAMILLES, RÉSEAUX. LES SUPPORTS SOCIAUX DE LA DIFFUSION DE LA SANTERÍA CUBAINE (CUBA-MEXIQUE)
}

\author{
Kali ARGYRIADIS *
}

La santería cubaine participe d'un processus de transnationalisation qui concerne l'ensemble des religions dites afro-américaines, présentes aujourd'hui en Afrique de l'Ouest, en Europe et sur tout le continent américain. Pour parvenir à cerner cet objet dans son ampleur, l'approche en termes de réseau semble a priori la plus opératoire. L'enquête ethnographique, quand elle concentre son attention sur les liens des acteurs, révèle un maillage complexe d'individus et de groupes reliés entre eux par un ou plusieurs intermédiaires. Mais peut-on se contenter de manière aussi abstraite du terme " réseau transnational » ? Derrière ce terme générique se dessinent en effet plusieurs réalités contingentes. À partir du cas havanais, puis de celui de la diffusion de la santería au Mexique, on tentera de préciser et de mettre à l'épreuve la pertinence de cette notion. [Mots clés : réseaux transnationaux, religion, santería, Cuba, Mexique.]

Ramas, familias, redes. Los soportes sociales de la difusión de la santería cubana (Cuba México). La santería cubana participa de un proceso de transnacionalización que abarca un conjunto de religiones llamadas afro-americanas, presentes hoy en dia en África del Oeste, en Europa y en todo el continente americano. Para poder analizar este objeto en toda su magnitud, el enfoque en términos de redes parece ser el más adecuado. La investigación etnográfica, cuando concentra su atención sobre los enlaces que unen a los actores, pone a la luz un complejo tejido de individuos y grupos ligados entre sí mediante uno o varios intermediarios. ¿ Podemos entonces conformarnos de manera tan abstracta con el término de "red transnacional »? En realidad, detrás de este término genérico se pueden observar varias realidades contingentes. Basándose en el caso habanero, y luego en el de la difusión de la santería en México, se intentará aquí precisar y poner a prueba la pertinencia de esta noción. [Palabras claves: redes transnacionales, religión, santería, Cuba, México.]

Ramas, families, networks. The social basis of the diffusion of Cuban santeria (Cuba, Mexico). Cuban santeria is following a global trend towards transnationalisation,

* IRD, UR 107 (Constructions identitaires et mondialisation), 23 avenue Henri Varagnat, 93140 Bondy, France [kali@argyriadis.net].

Journal de la Société des Américamistes, 2005, 91-2, pp. 153-183. O Société des Américanistes. 
like all of the other so-called Afro-American religions, currently found in West Africa, Europe and across the American continent. In order to grasp the full range of our topic, an analysis in terms of networks seems, at first glance, the best option. Detailed ethnography of the relationships between social actors reveals a complex ramification of individuals and groups connected by one or several intermediary links. However, such an abstract concept as " transnational network » is far from being fully satisfactory. Several contingent realities lurk behind this generic phrase, which we will attempt to refine and test by examining the situation in La Havana and the diffusion of santería in Mexico. [Key words : transnational networks, religion, santería, Cuba, Mexico.]

Depuis au moins une cinquantaine d'années, la santería cubaine ${ }^{1}$ s'est diffusée hors de Cuba, tout en recevant, même indirectement, l'influence de divers discours sur des pratiques religieuses très proches et se réclamant d'une origine yoruba, comme le candomblé nagô au Brésil ou le culte d'ifá au Nigeria. Â la circulation des idées, amplifiée par les moyens de communication modernes, s'ajoute la circulation physique des personnes. Artistes, chercheurs et intellectuels ont été, dans un premier temps, les principaux médiateurs reliant des espaces éloignés géographiquement. Les principales vagues d'émigration cubaines (au début des années 1960, en 1980 et au début des années 1990 jusqu’à nos jours) et l'ouverture de l'île au tourisme ont ensuite contribué à développer de façon significative ce phénomène. Aujourd'hui la santería est présente dans de nombreux pays voisins (États-Unis, Mexique, Porto Rico, Panama, Venezuela, Colombie...) et dans plusieurs grandes villes européennes (Paris, Madrid, Barcelone, Rome, Amsterdam...). Les pratiquants effectuent de nombreux déplacements entre ces différents lieux, privilégiant Cuba comme lieu d'initiation idéal, à moins qu'ils ne se détachent de la tutelle cubaine pour lui préférer celle des "Yoruba d'Afrique » ou d'autres modalités, s'inscrivant ainsi dans un réseau encore plus large.

La santería cubaine participe donc d'un processus de transnationalisation qui touche l'ensemble des religions dites afro-américaines, présentes aujourd'hui non seulement en Afrique de l'Ouest, au Brésil et dans la Caraïbe, mais également en Europe et sur tout le continent américain (Capone, éd. 2001-2002 ; 2004 ; Capone 2004). Pour parvenir à cerner cet objet dans son ampleur, l'approche en terme de réseau, et non en terme d'aire culturelle de type "Amériques noires » (Bastide 1967), semble a priori la plus opératoire ${ }^{2}$. L'enquête ethnographique, quand elle concentre son attention sur les liens des acteurs, révèle un maillage complexe d'individus et de groupes reliés entre eux par un ou plusieurs intermédiaires. Toutefois, malgré de nombreuses alliances ponctuelles (sans cesse en recomposition) et des discours insistant sur la cohésion et l'origine commune, ce phénomène reste fondamentalement acéphale, ou pluricéphale si tant est que ce terme conserve une cohérence au-delà d'une certaine quantité d'aspirants à une position d'autorité. Multilocalisé, il est traversé par des problématiques culturelles, 
" ethniques », " raciales » et politiques fortement ancrées dans leurs contextes nationaux respectifs. Mais ces différents ancrages locaux, qu'il convient d'analyser dans toute leur complexité, doivent être étudiés aussi dans leurs interactions mutuelles. Car, souvent, les ruptures et les rapprochements qui s'effectuent dans un cadre donné finissent par se répercuter à un autre bout du réseau. C'est d'ailleurs ce qui fonde cette notion selon Firth (1954, cité et traduit par Forsé 1991, p. 259) : " Par le terme de réseau, je ne veux pas simplement indiquer les "liens" entre les personnes; le terme de relation suffit à cela. Je veux plutôt indiquer qu'il y a liaison entre les liens eux-mêmes, ce qui a pour conséquence que ce qui arrive, pour ainsi dire, entre une paire de "nœuds" ne peut manquer d'affecter ce qui arrive entre une paire adjacente ». Les conflits et les rivalités, loin de remettre en cause le fonctionnement du réseau à grande échelle, contribuent en fait à son incessante reconfiguration.

Mais peut-on se contenter de manière aussi abstraite du terme de « réseau transnational » pour saisir les rapports entretenus au sein de la multiplicité des pratiques religieuses qui nous intéressent ? Derrière ce terme générique se dessinent en effet plusieurs réalités contingentes, qu'il convient de décrire afin de mettre à l'épreuve la pertinence de la notion. Un retour sur le terrain havanais s'avère essentiel pour contribuer à cette réflexion. En effet la pratique religieuse s'y organise sur le mode de la parenté rituelle, autour de «familles de religion » qui fonctionnent précisément comme des réseaux de relations et jouent souvent dans la vie quotidienne des pratiquants un rôle plus important que les parents consanguins ou les alliés matrimoniaux. L'individu utilise son appartenance à plusieurs de ces réseaux et négocie sans cesse sa position et ses liens au sein de ceux-ci. L'intégration de membres étrangers élargit leur champ d'action, mais qu'en est-il alors de l'efficacité et de la pérennité de ces réseaux hors de leur contexte d'émergence? Le cas de l'implantation de la santería au Mexique permettra de formuler quelques hypothèses afin de préciser la notion de réseau transnational de la santería cubaine.

\section{RAMAS ET FAMILLES RITUELLES HAVANAISES}

Reconstituer le réseau personnel d'un individu, " partir d'un ego et chercher ses liens avec d'autres unités » (Forsé 1991, p. 250), puis essayer de reconstituer un réseau de relations complet, comprenant l'ensemble des réseaux personnels connectés les uns aux autres (Degenne et Forsé 1994, p. 34), n'est pas une mince affaire. Les sociologues des réseaux de relations se sont heurtés aux limites d'une telle entreprise : « on serait vite confronté, du fait de la transitivité des relations, à un ensemble ingérable de données » (ibid., p. 30) et se sont résolus à opérer des découpages (classe d'école, entreprise, village) dont ils reconnaissent par ailleurs le caractère arbitraire. Je vais tenter ici, non pas de rendre compte de manière exhaustive de toutes les relations existantes à La Havane, mais de décrire un 
fonctionnement en réseau qui, par son caractère même, traverse et déborde de nombreuses frontières : celles de la parenté classique, mais aussi celles des appartenances sociales, institutionnelles, nationales et territoriales, sans pour autant remettre en question l'existence de ces dernières.

Les aléas et infortunes de la vie quotidienne conduisent souvent les Havanais à aller "se consulter ${ }^{3}$ auprès d'un spécialiste religieux recommandé par un proche ou une connaissance, voire par un médecin. L'offre est abondante et variée : les médiums spirites ${ }^{4}$ informent la personne sur l'identité des « morts de lumière donnés par Dieu » qui la guident tout au long de sa vie en échange de son attention ; les paleros ${ }^{5}$ la débarrassent des « morts obscurs » et autres sorcelleries envoyés par ses ennemis, puis lui proposent de conclure un pacte avec des esprits peu « évolués » qui travailleront pour elle ; les santeros (avec quatre morceaux de noix de coco ou seize cauris) et les babalaos (avec le système divinatoire appelé ifá), la renseignent sur les orichas ou santos qui l'accompagnent et qu'il faudra peut-être un jour recevoir ou, pour le principal appelé « ange gardien », fixer dans la tête. Les morts et les orichas eux-mêmes ne manquent pas de se manifester et de consulter l'assistance lors des nombreuses cérémonies publiques où, théoriquement, même les simples passants sont admis. Tous proposent des thérapies personnalisées et il est fréquent qu'un spécialiste d'une modalité de culte renvoie son interlocuteur à une autre modalité, à l'hôpital ou à l'église catholique, les messes étant par exemple considérées comme des dispositifs thérapeutiques efficaces.

C'est cette complémentarité fondamentale, doublée dans les faits d'un principe de cumul, qui conduit la plupart des Havanais à utiliser le terme générique de religión pour désigner et légitimer l'ensemble de leurs pratiques dans la conversation courante (Argyriadis 1999), eux-mêmes s'autodéfinissant comme " croyants » (creyentes) ou " religieux » (religiosos). Ce terme, fortement investi, dès la fin des années 1980, dans le contexte politique de réhabilitation des croyances religieuses de toutes obédiences, vient s'ajouter à celui de santería valorisé par les chercheurs cubains dans les années 1940 (Lachateñeré 2001 [1939]; Ortiz 1939) et cohabite aujourd'hui avec d'autres expressions comme ocha-ifá ou religión yoruba qui mettent, comme santería, l'accent sur cette modalité de culte considérée comme la plus prestigieuse, tout en insistant sur son caractère " africain traditionnel ».

L'intégration dans une famille rituelle commence souvent à partir du lien de confiance qui se crée entre un "consulté » et le spécialiste ${ }^{6}$ qui lui a donné satisfaction. Conseiller bienveillant, médiateur auprès des entités et thérapeute, ce dernier est peu à peu désigné par le terme de " parrain » ou « marraine ». Il ou elle « s'occupe de lui religieusement » (lo atiende religiosamente). Tôt ou tard, selon les cas, les protections et les « nettoyages ${ }^{7}$ ne suffisent plus à régler les problèmes du filleul. Par une cérémonie d'initiation, le spécialiste scelle de manière théoriquement irréversible le lien qui l'unit à son filleul - pour lequel il devient 
alors une sorte de nouveau père ou de nouvelle mère - et celui qui unit ce dernier à ses entités. Les autres filleuls deviennent pour leur part de nouveaux frères et sœurs, des "frères de religion » (hermanos de religión), issus de la même " branche » (rama), autrement dit du même lignage rituel ${ }^{8}$.

À quelques exceptions près, les spirites de la capitale ne pratiquent pas d'initiations à proprement parler. En revanche ils utilisent la terminologie du parrainage pour désigner les liens qu'ils entretiennent avec leurs "consultés » réguliers et les médiums qu'ils forment. Dans le palo, il existe trois stades initiatiques au moins : se rayer, devenir Padre ou Madre nkisa, devenir Tata ou Yaya nganga ${ }^{9}$ et ainsi pouvoir créer sa propre famille rituelle. Dans la santería, de nombreuses étapes préliminaires sont possibles, mais l'initiation à proprement parler consiste en la fixation de l'oricha principal ou « ange gardien » dans la tête du novice (asiento). Par la suite, le santero peut, entre autres, recevoir et donner d'autres orichas, parrainer des filleuls, franchir un deuxième grade initiatique lui permettant de réaliser des sacrifices (pinaldo) et se spécialiser dans la divination par les cauris (devenir oriaté). Un premier grade d'initiation à ifá est également accessible à tous (kofá pour les femmes; awofaká pour les hommes), mais les trois autres sont réservés aux seuls hommes hétérosexuels non sujets à la possession, ayant obtenu la permission de leur oricha dans le cas où ils sont aussi santeros.

À chaque nouvelle étape l'individu acquiert ou consolide ses liens avec de nouvelles entités, dont il se considère l'enfant ${ }^{10}$, mais qui « naissent » matériellement des entités de ses parrains et qu'il met à la disposition de ses frères de religion. Même lorsqu'un filleul réside à l'étranger, il arrive souvent qu'une partie de ses entités (leurs réceptacles) reste chez un parrain et continue ainsi à participer activement aux rituels, notamment en recevant les sacrifices et les offrandes et en se manifestant via un parent rituel possédé. Le filleul est alors censé en recevoir à distance les bénéfices. Les recommandations, avertissements et potins concomitants lui sont transmis par courrier, téléphone, internet ou personne interposée.

Les ancêtres rituels des parrains deviennent logiquement ceux du novice. Quant aux morts et aux orichas de chacun, ils sont indistinctement appelés " père » et "mère » et salués comme des membres de la famille. Les ancêtres biologiques sont rarement activés en tant que morts protecteurs. En revanche, chaque personne est accompagnée par un échantillon d'esprits de défunts, appelé « cordon spirituel», renvoyant aux origines "ethniques» présentées comme communes à tous les Cubains dans la Constitution et les livres d'histoire : il s'agit d'« Indiens ", d'Espagnols, de Congos, de Lucumíes " ${ }^{11}$, de Noirs créoles, de Mulâtres, de Gitans, d'Arabes, d'Haïtiens, de Français, de Chinois... La notion de famille rituelle peut donc être analysée sous différents angles et déborde déjà du simple cadre des liens entre parents rituels, interagissant par exemple avec le processus de construction identitaire nationale cubaine (Argyriadis 1999, p. 297) 
ou servant de schème d'interprétation des rapports sociaux (les enfants de tel oricha s'entendraient ou ne s'entendraient pas avec les enfants de tel autre).

Les représentations des relations parrains-filleuls sont calquées sur ce que devraient idéalement être les relations parents-enfants, c'est-à-dire des relations d'entraide, de confiance, d'affection mutuelle et de respect des plus jeunes pour les aînés. La hiérarchie au sein d'une famille de religion dépend du nombre d'années passées depuis l'initiation de chacun des membres et non de leur âge réel. Pour éviter certaines situations incongrues, il arrive souvent que l'on initie aussi (même symboliquement et en leur absence) le père, la mère ou l'époux du novice, afin d'empêcher que ce dernier bénéficie de plus de pouvoir, ce qui serait contraire aux idées selon lesquelles un enfant doit le respect à ses parents (oreja no pasa cabeza : l'oreille ne dépasse pas la tête) et une femme à son époux. Mais cette opération n'est pas systématique, surtout lorsque le novice est en conflit avec ses proches. En revanche, une mère ou un père biologique ne peut, en aucun cas, parrainer son enfant. Souvent, dans les familles de tradition religieuse, ce sont les oncles ou les tantes, voire les grands-parents ou encore un filleul de confiance de l'un des parents, qui assument cette charge.

Lignages rituels et lignages biologiques ne sont donc jamais équivalents, même s'ils se recoupent parfois. Mais c'est bien le vocabulaire de la parenté et des affects qui y sont liés qui prévaut dans la famille rituelle. Yolanda, une marraine santera, palera et spirite, parle de ses cinq filleuls de santo comme d'enfants supplémentaires et précise qu'«ils viennent pour n'importe quel problème, n'importe quelle situation, s'ils sont malades, n'importe quoi, moi je suis là, comme si j'étais leur mère. C'est une responsabilité que vous acquérez avec cette personne ". José Luis qualifie Rosa, sa marraine de palo et de santo de " seconde mère ", celle qui a " accouché de lui dans la religión » et à laquelle il doit un dévouement sans bornes. Dans son cas comme dans beaucoup d'autres, ce lien est tellement fort qu'il a pris le pas sur celui qui l'unit à sa mère biologique, provoquant dans un premier temps la jalousie de cette dernière, puis, en désespoir de cause, son rattachement en tant que filleule à la rama de Rosa : elle devient après coup, de son plein gré, la sœur aînée de son fils. En effet, lors de l'initiation de José Luis, elle avait été symboliquement initiée avant lui via quelques objets personnels la représentant.

Tous les filleuls n'investissent pas de façon aussi forte la relation au parrain ou à la marraine, ne serait-ce que parce qu'ils en possèdent souvent plusieurs. Un ou une spirite peut aussi être palero ou santero et " accoucher » de filleuls dans ces modalités de culte, mais tous ne peuvent accéder à l'initiation à ifá. Inversement, les babalaos ne peuvent parrainer que dans ifá. De plus, dans le palo, pour donner naissance à un novice, il faut obligatoirement un parrain et une marraine et, en santería, le parrain ou la marraine est accompagné d'un « second » (le sexe ici est indifférent), appelé ayubbón ou oyıgbona - terme traduit généralement par « les yeux qui te guident »-, qui sert de deuxième parent rituel ${ }^{12}$ et qui n'est pas 
forcément le même d'un filleul à un autre. Deux co-parrains (compadres) peuvent être également déjà inscrits dans un lien de filiation rituelle : souvent, lors de la toute première " mise au monde » qu'il réalise, le parrain ou la marraine prend pour co-parrain son propre parrain ou son oyugbona qui lui enseigne à cette occasion le déroulement de la cérémonie. Concrètement, chaque individu, outre qu'il peut se trouver lié à autant de ramas qu'il existe de modalités de culte, peut posséder plusieurs pères et plusieurs mères rituels. L'observation montre que chacun privilégie en général l'un d'entre eux en particulier, mais que cette préférence peut évoluer au cours du temps.

Autour d'un parrain ou d'une marraine, les filleuls sont, au fil des ans, plus ou moins assidus, plus ou moins présents, et le noyau de membres actifs change et évolue. Les relations entre frères et sœurs de religion sont moins profondes, moins soumises à des règles d'engagement affectif absolu que les relations parrainfilleul. Malgré un idéal d'harmonie affīché, elles peuvent être aussi bien cordiales que conflictuelles, sans pour autant que le lien rituel soit remis en question. En outre, dans les grandes familles (certaines peuvent atteindre plusieurs milliers de filleuls), tout le monde ne se connaît pas. L'un des devoirs censé être respecté par tous est justement d'assister aux anniversaires d'initiation ${ }^{13}$ ainsi qu'aux cérémonies publiques organisées par les uns et les autres, où l'affluence est un bon indicateur de l'état des relations, à ce moment précis, du commanditaire de la fête avec ses parents rituels.

De manière générale, de même que les sollicitations se font toujours par l'intermédiaire du parrain ou de la marraine, aucune initiative religieuse n'est prise entre frères sans passer par ce centre de gravité fondamental, qui fait figure de «point focal ", de pôle " où converge, par où transite l'ensemble des relations » (André 1987, p. 39). Cette terminologie qui emprunte à la notion de matrifocalité est particulièrement pertinente ici, notamment lorsqu'on admet, comme André, que par " mère focale », il ne faut pas entendre nécessairement " mère biologique ». L'insistance sur le rôle féminin prépondérant doit également être nuancée puisque dans plusieurs foyers cubains il existe des «pères focaux ${ }^{14}$ et que nombreuses sont les ramas dynamisées par un parrain.

Véritable agence de renseignements, le «point focal» de chaque famille rituelle consacre une grande partie de son temps, en dehors des consultations particulières, à mettre ses filleuls en rapport les uns avec les autres pour activer non seulement les échanges de " force ", d'aché ou de " lumière » nécessaires à la pratique religieuse, mais aussi les échanges de services indispensables à la vie quotidienne havanaise ${ }^{15}$. La plupart des ramas intègrent, quelle que soit leur taille, une variété de professions et de statuts sociaux très large qui permet, le cas échéant, à un professeur d'université d'obtenir rapidement un rendez-vous pour des analyses médicales (via tel frère de religion infirmier dans une polyclinique), à une femme de ménage de trouver un avocat pour son fils emprisonné (via une sœur de religion elle-même avocate), et ainsi de suite. 
Mais, surtout, intégrer une rama, quelle qu'elle soit, signifie pour un individu s'insérer dans un réseau de relations encore plus vaste, puisque chaque parrain est à son tour membre d'une ou de plusieurs familles rituelles. Enfin un initié peut à son tour créer sa propre famille en devenant parrain, en s'alliant à un ou, successivement, à plusieurs correligionaires. Maribel, l'une des filleules de Rosa en palo et en santo (mais sa sœur en ifá), qui est par ailleurs la femme de son jeune frère et vit sous son toit, comme José Luis, explique :

Dans le saint [santo] tout est une chaîne : il y a des parrains, des marraines, des oncles dans le saint, des tantes dans le saint, des grands-parents, des arrière-grands-parents de saint, toutes ces choses existent. Pourquoi ? Parce que les parrains de mon parrain, le parrain du parrain de mon parrain, tous ceux-là ont aussi à voir avec nous. Au final c'est une très grande famille. Mais une très grande famille où tout le monde n'a pas forcément de relations, parce que chacun vit sa vie dans sa maison, parfois nous nous rencontrons à un tambour, à une fête de religion, ou le jour où c'est l'anniversaire du parrain, où vont tous les filleuls... José Luis n'est pas mon frère de sang de religion, mais il l'est quand même : parce qu'il est fils d'Ochún comme moi ; mais c'est aussi le fils de saint de Rosa, et Rosa est filleule de mon ayubbón. Et alors mon ayubbón est filleul du parrain, de mon grand-père qui est le parrain de Rosa, et c'est par là que vient la chaîne. Quel que soit le côté par lequel tu la tires, la chaîne vient.

À cette multi-appartenance, liée au cumul des différentes modalités de culte et à une conception très élargie de la famille rituelle, s'ajoute dans les faits la possibilité de rompre les liens de parrainage et d'en former d'autres, généralement (mais pas toujours) au sein d'un même lignage rituel. Lorsque des conflits surgissent, le parrain est souvent accusé de négliger son filleul, de ne s'intéresser qu'à son argent, de ne pas lui transmettre suffisamment de connaissances rituelles ou de se comporter de façon trop autoritaire. L'initiation peut être qualifiée d'incomplète ou de mal faite. Enfin les parrains sont parfois soupçonnés d'avoir réalisé des travaux destinés littéralement à « subjuguer 》 ou « amarrer » le filleul, autrement dit de le tenir en son pouvoir ou de l'utiliser comme leurre. Amado, le co-parrain de Rosa, raconte :

Dans le palo, ça fait des années que je ne vois pas mon parrain. On s'est fàché, parce qu'il a voulu me tendre un piège, et mes morts, ma protection, ne l'ont pas permis. [...] Je suis Blanc, et il m'a commandé de faire un ebbó [offrande purificatoire] avec une poupée mulâtre, et lui il est Mulâtre. Il était déserteur du service militaire, et alors il m'a commandé de faire un ebbó pour que je me nettoie avec cette poupée et que je l'amène au poste de police. Alors je ne l'ai pas fait, ça allait retomber sur moi : la lettre c'est moi qui allait la prendre, la lettre de [le signe divinatoire qui évoque des problèmes avec la] justice. Et, quelques jours plus tard, il a été fait prisonnier. Et depuis ce temps, j'ai cessé d'aller le voir, je me suis séparé de lui.

En théorie, les liens parrains-filleuls sont inaltérables. Mais cette relation joue un rôle trop important dans la vie religieuse quotidienne pour pouvoir perdurer 
au-delà des tensions. Dans la pratique, si certains se contentent de l'éloignement ou de la rupture pure et simple, d'autres n'hésitent pas à changer de parrain ou de marraine. En palo, par exemple, il est possible de procéder à une sorte de ré-initiation, en remplaçant les scarifications rituelles par des tracés à la craie sur le corps du "novice » (rayar con tiza), en changeant son nom et sa signature rituels (pour que son ancien parrain n'ait plus d'emprise sur soi) et en reconstituant ses réceptacles à entités avec de nouveaux éléments. Dans la santería, il est courant de résoudre un conflit en finissant par s'adresser exclusivement à l'oyıgbona ou à un grand-parent de santo, situation qui peut même être entérinée par une cérémonie pendant laquelle, en présence de tous les intéressés et d'autres membres de la rama, la marraine ou le parrain rend à son (ou sa) filleul(e) les objets qui symbolisent leur lien ${ }^{16}$, à la suite de quoi ce dernier les remet alors à celui (ou celle) qu'il a choisi(e) pour nouveau parrain.

L'influence exercée par un parrain ou une marraine sur un filleul peut certes être considérable, notamment si leur relation affective est forte. Elle est cependant tempérée par sa non-exclusivité, liée au principe de multi-appartenance et à la possibilité pour tout filleul de se désengager de ce lien. Il convient de distinguer, en outre, l'influence de la coercition. À chaque occurrence consultatoire, les parrains (et de manière générale les consultants) proposent une série de conseils personnalisés censés émaner des entités. Ces conseils sont toujours variés, laissés à l'appréciation du consulté qui y opère des choix, décide de les ignorer ou les négocie. Les parrains ne donnent donc jamais d'ordres mais, pour reprendre leurs propres mots, des orientations toujours personnalisées. Enfin les quelques règles valables pour tous (respect envers les aînés en religion et respect entre membres, devoir moral de mise à disposition de sa force spirituelle ${ }^{17}$ ) n'impliquent en aucun cas le devoir d'obéissance. En dernier recours, c'est à ses propres entités, et à elles seules, que chacun se doit non pas d'obéir; mais de tenir les engagements pris lors des négociations précédentes. C'est une affaire personnelle dans laquelle un parrain ou une marraine ne peut s'immiscer qu'indirectement, en tant que médiateur, si toutefois son filleul fait appel à lui. La notion de respect en religión n'est en effet pas synonyme de déférence, mais de respect et de reconnaissance des particularismes des autres quels qu'ils soient (Argyriadis 1999, pp. 254-259).

Les aînés, par définition, ont des entités plus âgées, donc plus évoluées, plus savantes et/ou plus fortes : ce statut leur confère un réel prestige, doublé souvent d'un ascendant certain sur leurs filleuls. Toutefois, au sein d'une même rama, leur autorité n'est jamais absolue. Il existe toujours plusieurs référents, ne serait-ce que parce que les charges et les facultés rituelles ne peuvent jamais être condensées en une seule et même personne. " La famille rituelle est comme une forêt, et le savoir, comme la sève, coule dans toutes ses parties ", nous explique l'un des informateur de Menéndez (2002, p. 139). Les énergies (force, aché, lumière) de chaque être vivant et/ou de chaque élément naturel doivent être conjuguées et mises correctement en circulation pour qu'un rituel fonctionne. L'initiation 
requiert la présence de plusieurs « religieux » porteurs de compétences complémentaires : un maître de cérémonie à proprement parler (en santería il s'agira d'un oriaté, appelé aussi obbá), un sacrificateur, un parrain et/ou une marraine et/ou un oyugbona (pour " faire un saint »), un spécialiste des herbes, un ou une cuisinière et, pour le palo, des personnes ayant la faculté d'être possédées... Si plusieurs compétences peuvent être maîtrisées par une même personne, certaines sont complètement antagoniques par nature (par exemple être babalao ou joueur de tambour rituel et être possédé) ou dans un même contexte (être à la fois parrain et obbá, ou parrain et oyugbona).

Gobin (2003) a défini ce mode de fonctionnement en termes de principe de complémentarité ou d'interdépendance des rôles, s'articulant sur le principe de séniorité, a priori pyramidal. Les deux pôles organisent les relations d'alliance et de rivalité entre "religieux ", les positions de chacun se renégociant dans chaque contexte rituel (ibid., p. 53). Par exemple, un filleul peut être l'ainé en palo de sa marraine de santo ou, s'il est devenu oriaté, être son obbá et donc, ponctuellement, celui qui est censé lui donner les directives lorsqu'elle donne naissance à d'autres filleuls. Ajoutons à cela la dynamique introduite par la dimension de savoir et d'autorité rituelle incontestable (sur le moment) de la possession, par opposition à la connaissance acquise grâce au cumul des initiations et aux apprentissages qui en découlent. Dans le palo, où les morts se manifestent à chaque palier initiatique, elle est particulièrement forte et peut inverser complètement les hiérarchies lors d'un même rituel : ainsi une marraine, en " passant » un mort, peut se mettre à diriger les opérations, à sacrifier les animaux ${ }^{18}$ et à « consulter » l'assistance, s'attirant par extension la considération des filleuls au détriment de son co-parrain. Dans la santería, les compétences de certains acteurs religieux peuvent également être sérieusement mises en doute a posteriori par les orichas eux-mêmes (via les possédés), ébranlant la confiance des filleuls et contribuant à la reconfiguration de certains de leurs liens rituels.

\section{RAMAS, ASSOCIATIONS ET INSTITUTIONS NATIONALES}

Les relations au sein des ramas évoluent donc sans cesse, d'une part parce que la position de chacun y change régulièrement, d'autre part parce que la nature des liens entre membres est potentiellement transformable (d'alliance en conflit, de conflit en rivalité, de rivalité en alliance...), désactivable ou réactivable. Pour autant, ce principe évolutif ne remet pas en question le fonctionnement en réseau, qu'il faut entendre ici dans le sens d'une configuration en maillage et non dans celui d'une organisation dont les éléments, issus et subordonnés à une même origine, se répartiraient ensuite en différents points. Les familles de religion ne fonctionnent pas comme des communautés soudées, poursuivant un même objectif, mais plutôt comme des ensembles d'individualités reliées entre elles par des personnages qui leur servent à la fois d'axes et de relais (mais en aucun cas de 
leaders), et qui mettent en commun leurs capacités religieuses pour résoudre les problèmes personnels de chaque membre, et non des problèmes communs au groupe. À plus grande échelle, le réseau de ramas n'est pas dominé par un quelconque guide ou chef suprême, et ni la religión dans son ensemble, ni ocha-ifá en particulier (si tant est qu'à Cuba on puisse séparer cette modalité de culte des autres) ne comporte de dogme unique ou de commandements valables pour tous. Ce qui n'empêche pas, au contraire, de nombreux "religieux ", à différents niveaux, de tenter d'asseoir leur autorité et leur réputation sur un public qui déborde cette fois-ci largement le cadre de leur famille de religion.

Le fait en soi n'est pas surprenant, car la concurrence est rude pour attirer de nouveaux filleuls. Depuis l'ouverture de $1991{ }^{19}$, le champ « religieux » a été considérablement investi par les habitants de la capitale ; le nombre de spécialistes et de parrains potentiels s'y est multiplié de façon significative. Mais, au-delà de sa stricte dimension religieuse, ce phénomène doit être replacé dans un contexte social, politique et économique plus large. Aujourd'hui véritable atout de prestige, le fait d'être initié et d'appartenir à plusieurs ramas est également un facteur de réussite sociale. Il permet à chacun d'élargir et d'optimiser son réseau, alors même que l'utilisation des réseaux de relations est au cœur du fonctionnement de la société havanaise.

Les activités principales des hommes comme des femmes de la ville, outre le fait de se rendre sur leur éventuel lieu de travail, consistent à sortir pour " rendre visite » (visitar) et " voir si on résout quelque chose » (a ver si se resuelve algo). La politesse exige en effet, non pas d'inviter quelqu'un (à moins de prendre en charge son déplacement et sa nourriture), mais bien de lui rendre visite. On verra donc des gens traverser la ville malgré l'inconfort lié au manque de moyens de transport, dans le seul dessein d'entretenir leurs relations. Ils apporteront par exemple avec eux un peu d'ail (l'ail est arrivé à la bodega ${ }^{20}$ ) et repartiront peut-être avec un sac de tomates (dans cet autre quartier, ce sont les tomates qui sont arrivées, à tel point qu'on ne sait plus qu'en faire) et de précieuses informations : le nouveau fiancé de la fille de la personne visitée travaille dans une entreprise mixte important du matériel automobile, il peut vendre au noir et à moindre coût la pièce que le mari de la voisine, chauffeur de taxi privé, cherche désespérément depuis deux semaines.

Au cours d'une visite et pendant les trajets, on rencontre toujours de nouvelles personnes, elles-mêmes en visite, qui pourront avec le temps et le contexte devenir des relations principales. De gigantesques réseaux se forment, se défont et se recomposent ainsi, au gré des besoins. Les enfants, qui accompagnent régulièrement les adultes dans leurs pérégrinations, sont entraînés dès leur plus jeune âge à se souvenir des noms, des visages et, surtout, des fonctions qui les accompagnent. Ce ne sont pas seulement les liens de consanguinité, d'alliance matrimoniale ou de voisinage qui priment : ceux-ci sont au contraire souvent décrits comme source de conflits et cause des infortunes. L'herméneutique religieuse ne 
fait que renforcer ce sentiment. Lors des consultations, le thème du membre de la famille, voire du géniteur; qui a désiré la mort du consulté alors qu'il n'était encore qu'à l'état de foetus revient de façon récurrente et figure en très bonne place à côté de celui de l'époux(se), du voisin, de la voisine ou de l'ami(e) fidèle qui se révèle être l'instigateur de complots sournois et/ou de sorcelleries diverses.

Les réseaux de relations englobent en fait, au-delà du réseau de proches, toutes les rencontres faites au cours de la vie : anciens camarades de classe que l'on retrouve par hasard, collègues des emplois successifs, gens rencontrés lors d'une fête, ex-voisins et, bien sûr, membres de la famille de religion et/ou de l'organisation politique à laquelle on appartient éventuellement. À ceux-là viennent s'ajouter les relations qu'entretiennent ces derniers, le cercle s'élargissant et se recomposant ainsi à l'infini, pouvant déclencher un lien très éloigné géographiquement : par exemple, si la mère de l'un et le filleul de l'autre habitent la même ville à l'étranger, ils pourront se mettre en contact pour l'envoi de courrier et d'argent, sans devenir nécessairement proches. Beaucoup de migrants havanais qui résident dans des villes où ils sont très minoritaires ${ }^{21}$ continuent d'ailleurs à fonctionner selon une logique de réseau et répugnent à s'organiser en associations de type communautaire ou même à se côtoyer trop fréquemment sur le seul mode de la concitoyenneté. Ils décrivent cette façon d'être par l'expression « ensemble mais pas mélangés » (juntos pero no revueltos).

Il va de soi qu'une telle quantité de connaissances n'implique en aucun cas une amitié durable entre chacune des parties. Au contraire, les habitants de la capitale affirment n'avoir que peu ou pas du tout d'amis au sens strict du terme ${ }^{22}$. Tôt ou tard, les liens se déferont, le contexte n'étant plus le même. On préfère d'ailleurs en général le terme socio à celui d'amigo. Un socio, c'est une sorte d'associé face à une situation particulière : les relations sont fondées sur l'échange de services, de compétences (professionnelles, religieuses, intellectuelles...) ou de biens. Elles pourront bien sûr s'étendre au partage de moments de joie ou de tristesse, mais de la même façon que l'on se doit d'aider dans la mesure de ses capacités un inconnu qui se trouve brusquement confronté à un problème, ou de participer à une fête en manifestant son plaisir : elles correspondent aux attitudes considérées comme des règles de sociabilité de base.

Ce modèle de relations sociales pourrait être interprété, à La Havane, comme une conséquence directe du régime socialiste et on pourrait sans doute établir de nombreux points de comparaison avec d'autres capitales de pays régis par des systèmes politiques similaires, notamment en ce qui concerne la façon de se procurer des denrées en pénurie. Cependant, il a aussi été présenté par plusieurs auteurs comme caractéristique des zones urbaines de la Caraïbe en général. Benoist (1984, p. 78), s'appuyant sur les travaux de Foster (1963), remarque :

Tout se passe en effet comme si les liens sociaux qui se nouent aux Antilles prenaient de façon privilégiée la forme d'un contrat " dyadique », c'est-à-dire d'un accord direct et temporaire entre deux individus en vue d'une fin bien déterminée. [...] L'analyse des 
réseaux, exprimés ou latents, au sein desquels se font des contrats « dyadiques » est sans doute bien plus riche d'informations sur les véritables solidarités que le découpage du tissu social antillais en " communautés » fort peu réelles car, à travers une constellation de relations dyadiques, les individus s'articulent en réalité avec la société entière.

La notion de socio, quoi qu'il en soit, contraste fortement avec celle de compañero, érigée en modèle de sociabilité dans la Cuba révolutionnaire. En théorie tous égaux, le chef d'État y compris, unis par des liens de fraternité et de solidarité absolue, les citoyens cubains sont censés œuvrer ensemble pour le bien de la communauté nationale. Le terme compañero est abondamment utilisé pour l'ensemble des collègues de travail (mais il n'en gomme pas pour autant les hiérarchies, loin s'en faut) ainsi que dans les institutions politiques autorisées du pays : comités de défense de la Révolution, fédération des femmes cubaines, confédération des travailleurs cubains, union des jeunesses communistes... toutes organisées, comme le Parti communiste cubain, sur un modèle pyramidal (cellules, sections, comité central...). Cependant les compañeros, y compris ceux qui sont situés à d'autres degrés de la hiérarchie, jouent le plus fréquemment le rôle de socios hors du contexte professionnel ou militant - tant qu'ils ne sont pas désignés comme des ennemis jurés ou des rivaux, ou une fois le conflit passé, ce qui est facilité par la grande mobilité de travail et de résidence à La Havane.

Les réseaux de socios et les réseaux de ranas croisent donc sur leur chemin d'autres formes d'organisation sociale, en l'occurrence des institutions organisées hiérarchiquement, parmi lesquelles, et non des moindres, celle qui à Cuba englobe toutes les autres: l'État-nation. L'émergence depuis une quinzaine d'années d'associations ou de groupements religieux ayant pour principal objectif l'orthodoxisation de la santería et d'ifá n'est pas étrangère à cette confrontation. Dans le contexte de crise politique et économique liée à la disparition de l'Union soviétique, le gouvernement cubain a cherché explicitement, dès la fin des années 1980, de nouveaux interlocuteurs et guides religieux alliés sur les questions d'éthique et de renforcement du sentiment communautaire (Argyriadis et Capone 2004, pp. 83-88). Outre de nombreuses alliances stratégiques successives avec un large éventail d'églises et de mouvements chrétiens (théologiens de la libération, pasteurs pour la paix, patriarche orthodoxe de Constantinople...), il a contribué à la création, en 1991, de l'Association culturelle yoruba de Cuba, dont l'hégémonie institutionnelle a été rapidement mise en cause par la constitution de groupes rivaux (ibid., pp. 89-95).

Les luttes de pouvoir entre "religieux » sont aujourd'hui nourries par une nouvelle catégorie d'accusation : celle d'être à la solde de l'un des deux camps politiques qui s'affrontent actuellement (anti ou pro-Castro). Les précédentes marquaient déjà l'inscription de ces pratiques sur la scène publique : celle de sorcellerie malfaisante pour se positionner dans un contexte de diabolisation de l'africanité, celle de mercantilisme et celle de non "traditionnalité » pour s'affĩrmer au sein d'une société qui commençait à reconnaître une valeur à 
l'origine culturelle yoruba, les trois coexistant de nos jours avec la quatrième et interrogeant indirectement la notion de cubanité, alors même que la santería cubaine se transnationalise (ibid.). Les nouvelles associations religieuses havanaises poursuivent donc les stratégies de légitimation engagées depuis au moins deux siècles par d'autres types de structures ${ }^{23}$, déterminées à chaque fois par leurs cadres politiques d'émergence. En cela, leur situation ne serait pas différente de celle d'autres réseaux étudiés par la sociologie des mouvements religieux: « contestataires d'institutions dont ils rejettent la loi, ils sont tout de même contraints, pour exprimer leur protestation, d'intégrer dans le fonctionnement de leurs organisations certains des traits et des valeurs qu'ils réfutent » (Colonomos 1995, p. 44).

Ainsi l'empire colonial espagnol a longtemps permis aux esclaves, aux affranchis et à leurs descendants de se constituer en associations d'entraide, tout comme les colons, à condition que ces cabildos soient conçus sur le modèle des confréries religieuses, sous l'égide d'un saint patron ${ }^{24}$. Leur rôle dans les luttes antiesclavagiste et indépendantiste devenant par trop évident, de nombreuses mesures administratives furent prises pour empêcher leur fonctionnement, notamment pendant les guerres d'indépendance. Transformés peu à peu en sociétés de divertissement et d'entraide, beaucoup disparurent complètement au début du $\mathrm{xx}^{\mathrm{e}}$ siècle (Ortiz 1992 [1920]; à propos de leur survivance dans certains contextes, voir Testa 2005). Mais l'intérêt des chercheurs cubains pour leurs derniers avatars à partir des années 1920, puis leur valorisation en tant que modèles de résistance anti-colonialiste aux débuts de la révolution, contribuèrent à la conservation de certaines d'entre elles et à la création de nouvelles sociétés et associations parmi lesquelles seules les plus engagées aux côtés de l'État purent maintenir un statut d'interlocuteurs pendant les années 1970, où les pratiques religieuses en général et afro-cubaines en particulier n'étaient tolérées que par le biais de leur expression esthétique (Argyriadis 2006).

Il serait tentant de voir dans ces différentes organisations culturelles et religieuses des formes institutionnalisées de familles de religion. S'il est fort probable que les cabildos et les sociétés d'entraide ont été en premier lieu des ramas, il n'en va pas de même pour les associations contemporaines. En effet, leurs membres n'appartiennent pas aux mêmes ramas, dont les parrains seraient les leaders. Certes, les discours destinés à leurs interlocuteurs étrangers donnent l'impression qu'il s'agit de « communautés » religieuses prenant la forme d'associations informelles, notamment en mobilisant la référence à la généalogie rituelle et consanguine ${ }^{25}$. Toutefois, sur le terrain, on s'aperçoit très vite que le fait d'être le filleul d'un leader d'association n'engage en rien l'appartenance à cette dernière, ni même le partage d'idées communes en matière de traditionnalité ou de stratégie politique.

Au sein d'une même rama, on trouve des filleuls qui choisissent de s'affilier ou de prendre parti pour l'un ou l'autre de ces groupements, voire par pragmatisme 
explicite pour plusieurs à la fois (certains les appellent alors, par dérision, « les agents doubles »). Plus couramment encore, la majorité des " religieux » préfèrent ne s'affilier à aucun d'entre eux. Inversement, au sein d'une association, on trouve des membres issus de ramas très diverses. Leurs leaders même ne dérogent pas au principe de multi-appartenance: au jeu des rivalités et des alliances recomposées s'ajoute le fait que les concurrents les plus acharnés peuvent faire partie de la même famille de religion. C'est le cas, par exemple, de Guillermo Diago, babalao, membre de la commission organisatrice de la «Lettre de l'année » qui, chaque 31 décembre, prépare la cérémonie prétendant à la production de prévisions valables pour l'ensemble des religieux. La « Lettre de l'année » de la commission, rivale de celle de l'association culturelle yoruba de Cuba, est présentée comme "la plus authentique», car non soupçonnée de tentative de manipulation par l'État. Mais Guillermo Diago est aussi le filleul en ifá d'un défunt membre fondateur de l'association, Ángel Padrón Cárdenas, et le frère cadet rituel de son numéro deux actuel, Omar Quevedo. En total désaccord d'un point de vue institutionnel et idéologique, ils n'en restent pas moins frères de religion et se retrouvent inévitablement dans des rituels auxquels ils participent ensemble dans le plus grand respect, à défaut de cordialité.

Les associations religieuses contemporaines et les réseaux de ramas ne se situent donc pas du tout sur le même plan. Les premières sont constituées de membres certes liés par une pratique religieuse commune, mais pas nécessairement par des liens de parenté rituelle. Les seconds connectent entre eux tous les religieux, quelles que soient leurs opinions et leurs prises de position. Les associations sont portées par un projet et des leaders communs, et disparaissent lorsque ces derniers perdent leur capacité à rassembler ${ }^{26}$. Mais les luttes de pouvoir au sein du réseau de ramas contribuent à en constituer d'autres, tant que le contexte politique le permet, étant donné leur fonction de «mises en scène publiques du champ religieux » (Gobin 2003, p. 9) et de médiation avec les institutions nationales et internationales. Portées par des dirigeants qui parfois, à défaut d'être des parrains reconnus, sont capables de médiatiser leurs discours et leurs actions et de passer aisément du registre religieux aux registres culturel, artistique, économique et politique, les associations permettent d'engager une relation efficace (en terme de captage de ressources symboliques, politiques et/ou financières) avec l'État et ses institutions ainsi qu'avec les ONG, les universités et les associations religieuses "africaines » ou "afro-américaines » qui se sont multipliées dans d'autres pays. La participation et l'organisation de colloques, conférences, festivals et rencontres diverses rendent notamment possible l'accès à un débat à l'échelle transnationale sur la « tradition yoruba » ou sur la question cruciale de la reconnaissance de cette même tradition comme religion universelle fondatrice de toutes les marques de "civilisation » : monothéismes, science et médecine moderne, grands courants philosophiques... 


\section{RaMAS TRANSNATIONALES, RAMAS RELOCALISÉES}

À La Havane, les réseaux de socios (« dyadiques » et acéphales) combinés avec les réseaux « religieux » (multicentrés) traversent l'ensemble de la société, en se connectant en de multiples points avec les institutions nationales et internationales et en interagissant avec elles de façon médiatisée, via les institutions-portes que sont notamment les associations religieuses. Permettant de résoudre individuellement les problèmes quotidiens et de contourner grand nombre de règles et lois, ils débordent d'une certaine manière les catégories et hiérarchies sociales, de même qu'ils franchissent très vite les frontières de la ville et du pays. Cette transversalité ne remet cependant pas en question l'existence des institutions, catégories et frontières. Elle contribue plutôt à leur évolution et à leur reconfiguration.

L'évolution de la santería est, par ailleurs, depuis longtemps, inscrite dans un contexte que l'on peut qualifier de transnational, ne serait-ce que parce qu'elle se nourrit, depuis l'arrivée des premiers esclaves et des premiers colons à Cuba, d'un rapport ambivalent à deux référents culturels : "l'Afrique » et " l'Europe ». Et c'est bien parce que des élites intellectuelles et artistiques formées en Europe découvrent et valorisent "l'Afrique » au début du $\mathrm{xx}^{\mathrm{e}}$ siècle qu'elle sort de la marginalité et conquiert progressivement un statut relativement légitime dans l'île (Argyriadis 2006). Les réseaux de ramas havanaises, qui se tissent à partir de chaque nouveau membre et se déploient en amont, en aval et horizontalement, ont suivi cette progression et se sont translocalisés à mesure que les pratiques religieuses sortaient de la clandestinité. Plusieurs «lieux» se disputent aujourd'hui le caractère de plus haute ancestralité et authenticité traditionnelle yoruba, au fil des récits lignagers des ramas, mais aussi de l'intérêt des chercheurs pour ces derniers, lequel vient parfois leur conférer un statut de vérité historique : les quartiers de Regla, Guanabacoa et Marianao à La Havane, Matanzas et Palmira dans l'île, pour ne citer que les plus couramment mentionnés. Le recrutement des filleuls se fait bien au-delà des limites du quartier et de la ville des parrains : le bouche à oreille à travers les réseaux de relations, la réputation et la satisfaction personnelle sont des facteurs de choix bien plus importants que la proximité géographique.

Les vagues d'émigrations dans un premier temps, puis l'ouverture du pays au tourisme ont prolongé logiquement l'expansion des réseaux de famille de religion hors du territoire national, qui semble s'être amorcée dès les années 1950 en suivant justement les parcours et voyages de différents artistes (pour les ÉtatsUnis voir notamment Capone 2005, pp. 93-94; Guedj 2004, p. 31 ; pour le Mexique voir Juárez Huet 2004, p. 63). En 1991, la présence d'étrangers dans la parenté rituelle restait néanmoins occasionnelle à La Havane, mais elle s'est accrue de façon saisissante dans les années qui ont suivi. Aujourd'hui, non seulement il est devenu rare de rencontrer un parrain ou une marraine qui n'ait 
aucun filleul étranger ou habitant à l'étranger, mais de surcroît, parmi eux, nombreux sont ceux qui ont été invités "à l'extérieur » pour participer à des rituels et garantir ainsi l'authenticité de ces derniers par leur simple présence, voire pour prendre la parole lors de conférences, colloques et festivals ayant pour thème les cultures « africaines » ou " afro-américaines 》 ou encore l'ésotérisme ou l'alter-mondialisme. À travers ces allers-retours, ils activent des liens qui débordent à nouveau le strict champ religieux et deviennent acteurs de réseaux qui traversent les trois régions qui les fondent historiquement : les Amériques, l'Europe de l'Ouest et l'Afrique de l'Ouest, dans un contexte et des rapports de force qui ne sont évidemment plus les mêmes que par le passé.

La question qui se pose alors est de savoir si, déployés dans un espace aussi vaste, les réseaux de ramas dont on parvient à comprendre le fonctionnement à l'échelle d'une ville se reproduisent à l'identique ou s'ils se transforment tout au moins localement, s'ils « s'indigénisent » (Appadurai 1996, p. 67). Autrement dit, que se passe-t-il quand, en " tirant sur la chaîne », on rencontre des parents rituels avec des références culturelles, religieuses et identitaires très éloignées les unes des autres ? L'ancrage d'un réseau en de multiples localités éprouve-t-il sa solidité d'ensemble ? L'exemple de l'implantation de la santería cubaine au Mexique offre de nombreuses pistes pour répondre à ces interrogations.

L'arrivée des pratiques « religieuses » cubaines dans ce pays semble dater des années 1950. Elle s'effectue dans le sillage des intenses échanges artistiques entre Cuba et le Mexique, puis dans celui des flux migratoires qui passent par les États-Unis. Une progression plus nette se dessine dans les années 1990, liée au contexte d'ouverture de l'île (Juárez Huet 2004, p. 67). Toutefois, la santería reste numériquement faible ${ }^{27}$, de même que d'autres mouvements religieux émergents (comme les pratiques néo-ésotériques ou néo-indigénistes...), et plus de $90 \%$ des Mexicains s'affirmeraient toujours comme catholiques. De nombreux auteurs s'accordent cependant à reconnaître le caractère syncrétique et pragmatique de ce " catholicisme populaire ", terreau propice et toile de fond à la cohabitation entre des pratiques a priori extrêmement éloignées les unes des autres (voir à ce sujet de la Torre et Mora 2001). Comme à Cuba, pratiquer la santería et aller à l'église catholique sont deux choses tout à fait compatibles.

Ce qui frappe, notamment quand on se penche sur les réseaux " religieux » essentiellement cubano-mexicains (d'autres nationalités peuvent aussi être présentes), c'est que la dimension identitaire nationale intervient comme nouvel élément nourrissant les luttes de pouvoir. Ce point a été relevé par Juárez Huet (2004, p. 76) à propos des santeros mexicains dont les parrains ou les grandsparents rituels sont des Cubains immigrés :

Alors que les Cubains sont actuellement les plus avancés dans la hiérarchie et que plusieurs sont reconnus et respectés pour leur savoir, beaucoup de Mexicains jettent sur eux le discrédit, arguant du fait que ce sont eux qui créent le désordre et commettent des méfaits, que « ce sont de bonnes personnes chez eux, à Cuba, mais quand ils viennent au 
Mexique, [...] ils ont recours aux magouilles et aux tromperies pour soutirer l'argent qu'ils ne peuvent pas gagner chez eux »; " certaines personnes pensent que la santería étant d'origine cubaine, c'est seulement à Cuba qu'on trouve ce qu'il y a de mieux, mais ils se trompent, parce que nous, les Mexicains, ne demandons rien aux Cubains ». C'est ainsi que les Mexicains tentent de légitimer le savoir qu'ils détiennent aujourd'hui dans la santería.

De fait, la prolifération des initiations faites aux étrangers ne s'accompagne pas d'un traitement identique pour les filleuls cubains et les filleuls non-cubains. À La Havane, les prix sont multipliés par dix en moyenne, et au moins par deux ou trois pour les « Cubains de l'extérieur », catégorie intermédiaire problématique qui a surgi récemment, alors que ces derniers reviennent de plus en plus régulièrement chez eux pour passer des vacances et pour s'initier ou effectuer des cérémonies qu'ils ne peuvent pas faire dans les pays d'accueil ${ }^{28}$. Les Cubains de l'île justifient cette inégalité par la conviction que tout visiteur étranger a un niveau de vie supérieur au leur ${ }^{29}$ et qu'il va recevoir un service rituel de haute qualité, puisque de tradition cubaine. Toutefois, ils jugent d'un œil critique cette pratique de gonflement des prix lorsque c'est un parrain étranger qui l'applique à un futur initié originaire de son propre pays. Une oyugbona cubaine s'indigne par exemple à propos de sa co-marraine mexicaine qui empoche un confortable bénéfice lors de l'initiation d'une filleule « de sa propre race ». Pour démontrer par contraste son honnêteté, elle évoque ses filleuls mexicains, colombiens et panaméens en précisant : « eux je les traite comme mes filleuls cubains ».

L'idéal de dévouement identique pour tous les filleuls, réaffirmé ici avec d'autant plus d'insistance que la chose ne va pas de soi, est donc contrebalancé par le principe implicite de solidarité ou de préférence nationale. En situation de migration, le phénomène semble perdurer. Ainsi, un santero, palero et spirite cubain, vivant à Mexico, qui règne en maître absolu sur un public de filleuls mexicains subjugués et se met en scène en tant que tel pendant les rituels, adopte une tout autre attitude avec les quelques participants cubains de passage dans ses cérémonies. Sans nécessairement les connaître ni même s'assurer qu'ils sont santeros, il les accueille avec le terme d'adresse " abure », qui signifie frère de religion au sens large. Un autre explique à ses concitoyens, sur un ton confidentiel, qu'il a décidé de ne pas remettre de réceptacles à entités à ses filleuls de palo mexicains dès le premier grade d'initiation car " ils pourraient en faire mauvais usage ». Accessoirement, ce geste leur coupe l'accès à l'apprentissage rituel et à la communication directe avec leurs morts, les condamnant à dépendre de leur parrain en toute circonstance.

Les stratégies développées par les Mexicains pour contourner cette inconfortable position d'éternels subordonnés sont comparables à celles observées dans d'autre pays « d'accueil » de la santería cubaine (Argyriadis 2001-2002; Capone 2005) : suite à ce qu'ils désignent comme une prise de conscience, certains se raccordent aux mouvances rivales, notamment celle des Yoruba du Nigeria, 
appelée tradicionalismo au Mexique, à laquelle ils ont accès via les États-Unis ${ }^{30}$. À l'idée d'une suprématie cubaine en matière de tradition, ils rétorquent donc que si l'appartenance ethnique ou nationale joue un rôle primordial dans cette religion, alors les Cubains doivent accepter la souveraineté des Yoruba nigérians qui en sont à l'origine. Certains santeros originaires de Veracruz poussent la logique jusqu'à préciser que, puisque les santeros cubains ne le font pas, ils doivent alors admettre n'être qu'une variante parmi d'autres et reconnaître les autres variantes possibles, notamment la variante " mexicaine ». Cette dernière désigne une manière d'établir un lien, via le palo et le rapport aux esprits de défunts, avec certaines pratiques locales (désignées par le terme brujeria, « sorcellerie ») considérées comme proches des pratiques afro-cubaines par les « religieux » locaux comme par les chercheurs partisans de la reconnaissance de l'apport culturel africain au Mexique (voir, à ce propos, l'article d'Hoflimann dans ce même numéro). Ainsi, leur "cordon spirituel» se trouve enrichi de prêtres mayas, olmèques ou aztèques et d'anciens « sorciers » de la région, tandis qu'ils ancrent localement leur légitimité par une référence à un ou une aïeule "sorcière ». Les rapports de force s'en trouvent considérablement transformés, non seulement sur leur lieu de résidence et d'exercice, mais aussi à La Havane (dans les contextes rituels spirites et paleros) où la possession et la voyance sont prises très au sérieux en tant que source directe de connaissance.

Ces différents " raccordements » témoignent du déplacement de la logique dynamique interne à un réseau de ramas (transferts de tutelles pour affirmer ou acquérir une position d'autorité) sur une échelle plus large, où s'affrontent plusieurs courants religieux dont l'ancrage local de départ est débordé pour finir par nourrir des constructions identitaires extrêmement éloignées. Ici c'est bien le " recours à un élément tiers » qui permet de fonder sa propre identité (Amselle 2001, p. 7). Si, sur ce point, au Mexique, le fonctionnement en réseau reste opératoire, il semble qu'il n'en va pas de même de l'évolution de la nature des relations au sein de chaque famille de religion. Celle-ci est revisitée, tant par les Cubains implantés au Mexique que par les Mexicains. Forts d'un prestige parfois mêlé de crainte, pour l'instant peu soumis à la concurrence, les santeros les plus âgés rituellement, quelle que soit leur nationalité d'origine, nouent des rapports nettement plus hiérarchisés avec ceux qu'ils appellent parfois leurs " disciples ». Le terme rama lui-même finit par être employé pour évoquer exclusivement le lignage rituel du locuteur, tandis que certains désignent souvent la famille de religion qu'ils ont fondée comme une " communauté ».

En effet, le discours des " religieux » de Mexico et de Veracruz autour des notions de famille et de respect diffère considérablement de celui de leurs homologues havanais. La famille (au sens classique du terme) est systématiquement placée au centre de leurs préoccupations : beaucoup "se consultent " pour résoudre, non pas un problème individuel, mais un problème qui touche l'un de leurs parents (père, mère, frères et sœurs, conjoint, enfants, grands-parents, petits- 
enfants, oncles et tantes, cousins germains...), considéré comme autrement plus grave et source de souffrances bien plus difficiles à supporter. La famille est en outre sans cesse sollicitée comme modèle communautaire et référence de base, dont le père ou le frère aîné est le chef incontesté, forçant le respect (respect et obéissance aux injonctions étant ici clairement synonymes). L'initiation d'un Mexicain entraîne généralement l'implication "religieuse » d'une bonne partie de sa famille, tandis qu'à l'inverse la réprobation familiale semble freiner son engagement. Un santero de Veracruz témoigne :

Il y a des cas de frères de religion qui se sont initiés, puis se sont séparés parce qu'ils avaient des problèmes avec des membres de leur famille ou avec leur conjoint [...] ils se sont sans doute dit : pourquoi perdre ma famille pour quelque chose que je commence à peine?

Beaucoup de ramas dont le parrain est mexicain ont d'ailleurs pour membres essentiellement les membres de la famille consanguine de ce dernier (appelés parientes carnales, parents de chair). Juárez Huet (2004, p. 75) remarque à ce propos :

Parenté rituelle et parenté sanguine sont souvent interdépendantes. Il existe des familles où un frère devient le parrain de ses frères et sœurs, ou même de ses neveux et cousins. [...] Les parrains et marraines qui sont unis par des liens à la fois consanguins et rituels ne sollicitent pas seulement l'intervention des saints ou des oracles pour essayer de modifier la conduite d'une personne. Ils s'appuient également sur les liens affectifs, sentimentaux, et sur l'autorité qui découle de leur position dans la parenté consanguine, position qui se trouve ainsi renforcée par leur double rôle d'aînés.

L'étude des « quiproquos culturels » et de leurs conséquences sur l'évolution de la pratique religieuse des uns et des autres reste à approfondir dans les cas, très nombreux, où une rama intègre des membres de plusieurs nationalités, et notamment des Cubains et des Mexicains. Ceux qui concernent la notion de famille sont légion. L'exemple de Pedro, un Mexicain trentenaire qui a «fait son saint » à Cuba depuis deux ans, illustre bien ces décalages. Pedro a découvert la santería par le biais d'un babalao mexicain, Raúl, qu'il considère toujours comme son parrain principal. Celui-ci lui a fait passer à Mexico un premier stade initiatique en ifá et en santería, ainsi qu'à son épouse, son fils et ses deux frères. Il l'a aussi emmené à La Havane où Pedro s'est d'abord rayé en palo (il s'est disputé ensuite avec son parrain cubain), puis lors d'un deuxième voyage a "fait son saint » avec Emilio, l'un de ses frères aînés d'awofaká, cubain également. Ce dernier, leader d'une association informelle prônant l'orthodoxisation du corpus de textes liés à ifá, fonde sa stratégie à l'égard de ses interlocuteurs étrangers sur une présentation du groupe en tant que communauté familiale unie : les membres sont, entre autres, ses frères et sœurs et leurs enfants, vivant tous sous le même toit. Plus tard, Emilio fait également leur saint à la femme de Raúl et à l'une de ses amies. 
Pendant la cérémonie, Pedro et Emilio se brouillent, notamment parce qu'Emilio remet trop souvent en question l'autorité rituelle des babalaos présents, et celle de Raúl en particulier. Pedro présente les raisons de son éloignement avec ses deux parrains cubains successifs comme une réticence de sa part face à leur « instabilité familiale ». Ce qui, à Cuba, est une preuve relativement extraordinaire de cohésion (les frères et sœurs habitent ensemble alors que les géniteurs sont décédés) n'est pas perçu de la sorte par Pedro qui se dit très choqué du fait que les sœurs soient divorcées ou vivent en concubinage, pire, que l'une des nièces ait deux enfants sans être mariée et sans vivre avec leur père. Pedro aspire à devenir très vite babalao et ainsi « religieusement indépendant » : aux côté de son épouse et de ses frères initiés par lui dans d'autres charges rituelles essentielles (mais subalternes), il envisage de fonder une famille de religion qui ne sorte pas du cercle de ses proches, dont l'activité ferait vivre toute sa famille et dont il serait bien sûr le chef incontesté. Il commence en outre à s'intéresser à la littérature « traditionnaliste » yoruba dont il a pris connaissance grâce à internet.

À l'instar de plusieurs autres santeros exerçant au Mexique, Pedro utilise, d'une part, comme à La Havane, la stratégie de rupture avec les parrains et d'activation de nouvelles alliances y compris hors du champ de la rama: le fonctionnement en réseaux à l'échelle transnationale reste totalement investi de ce point de vue. Mais, d'autre part, pour consolider une position à l'échelle locale, il semble que s'opère peu à peu une transformation des familles-réseaux en « communautés » rituelles consolidées par les liens de parenté consanguine, où les rapports d'autorité ne sont plus les mêmes et où l'accès au réseau de ramas et par extension à la connaissance rituelle devient, tout au moins dans un premier temps, le privilège exclusif du parrain ${ }^{31}$. En témoigne également la façon dont s'effectuent certaines initiations de Mexicains à La Havane, où les novices, sous prétexte d'être protégés de l'avidité de religieux malhonnêtes, sont confinés par leurs parrains sur le lieu de la cérémonie et fortement découragés dans leurs tentatives d'engager la conversation avec les personnes présentes. Une enquête de plus longue durée est nécessaire pour mesurer l'efficacité de ces méthodes et la stabilité de ce modèle.

L'approche en terme de "réseaux transnationaux » et non en terme de " communauté transnationale » peut fournir de nombreux outils pour appréhender le processus de diffusion sur trois continents des religions censées être d'origine yoruba (notion qui génère à la fois les possibilités d'alliance et les dissensions internes), à condition de préciser les termes à partir de l'ethnographie. L'exemple de La Havane, prolongé ici dans ses extensions mexicaines, montre d'abord que les réseaux constituent des formes d'organisation sociale qui, loin de remettre en question la solidité des institutions qu'ils côtoient, contribuent d'une certaine manière à leur évolution et à la formation de certaines d'entre elles. Pour Granovetter (2000, p. 45), comme pour d'autres sociologues des réseaux de 
relations, l'analyse des processus qui se développent dans les réseaux interpersonnels permet de « relier de manière convaincante les interactions micro-sociales et les phénomènes macro-sociaux $»$.

L'un des problèmes qui se pose justement dans le cas des réseaux « religieux » est celui de la définition des échelles. Car, en intégrant une ou plusieurs ramas, chaque pratiquant s'insère dans deux types d'espaces d'échanges qui ne s'embô̂tent pas forcément palier par palier, mais qui interagissent entre eux : un espace territorial, avec ses échelles locales et nationales spécifiques et l'échelle tricontinentale dans laquelle évolue pour l'instant le réseau de la " religion des orisha »; et un espace « relationnel » où la famille de religion constitue l'échelle " microsociale » pour ses membres, alors qu'elle peut déjà déborder toutes les limites territoriales et en ce sens être qualifiée de « transnationale ». Certes, les exemples décrits ci-dessus témoignent de l'importance de la dimension nationale, hors de laquelle les modalités de l'échange et les rapports de force se transforment. Toutefois, outre le fait que de nombreux liens - positifs et négatifs - sont activés malgré l'éloignement géographique, la prise en compte de toutes les relations (de la «force des liens faibles » pour reprendre la théorie de Granovetter 2000), y compris celles ponctuelles, indirectes et même virtuelles ou imaginaires, s'avère nécessaire pour comprendre les initiatives prises par chacun des acteurs du réseau et la façon dont circulent les individus, les idées, les symboles et les objets.

L'analyse ethnographique a montré que les réseaux de ramas peuvent se combiner avec des groupes institués inscrits dans des problématiques fortement localisées ou « re-localisées ». Dès lors, face à ces associations (il en existe dans tous les pays où la santería est présente, et au moins deux au Mexique) dont les leaders détiennent l'essentiel des compétences en matière de captage de ressource et concentrent sur eux toute l'attention médiatique, on peut se demander si la notion de réseau reste pertinente. Le paradoxe fondateur entre la plasticité de pratiques intégrées dans un réseau de relations acéphale, d'une part, l'aspiration à l'unification et à l'« orthodoxisation », d'autre part, remarqué par plusieurs auteurs pour les religions dites afro-américaines en général (Argyriadis et Capone 2004, p. 125) semble rejoindre le paradoxe de la présence des réseaux dans l'espace socio-politique souligné par Colonomos (1995, p. 173) :

Trop visibles, ils risquent de s'institutionnaliser et de devenir statiques en empêchant ainsi la formation de nouvelles alliances ; trop opaque, leur clandestinité les éloigne de l'accès à la parole à l'intérieur du système et freine la vocation pluraliste ou plurifonctionnelle qui fait leur force.

L'erreur consiste sans doute à confondre entre elles des configurations qui sont en réalité différentes et n'ont pas la même fonction. Pour Colonomos (ibid., p. 22), un réseau est une "organisation sociale composée d'individus ou de groupes dont la dynamique vise à la perpétuation, à la consolidation et à la progression des activités de ses membres dans une ou plusieurs sphères socio- 
politiques », définition qui s'applique assez bien aux associations religieuses, créées sur l'initiative de quelques personnes qui se trouvent en position de force dans un contexte donné, mais ne sont en aucun cas des familles de religion qui se transformeraient sur un mode institutionnel. En revanche, ce sont bien ces associations qui servent de relais entre le réseau des ramas et l'État, et qui permettent également de contourner certaines contraintes de ce même État par l'inscription dans des réseaux transnationaux. Mais l'insertion dans un espace de relations qui déborde le cadre local est d'abord réalisée à l'échelle de chaque famille de religion, dont la logique de fonctionnement permet de connecter entre eux des individus très éloignés socialement, géographiquement et culturellement, tout en générant à l'infini de nouvelles branches qui peuvent se re-territorialiser, sans jamais perdre complètement leurs liens aux autres familles de religion ou aux autres groupes par rapport auxquels elles se positionnent via l'affirmation d'une filiation, d'une alliance ou d'un antagonisme.

C'est peut-être là toute la diffǐculté qu'il y a à saisir le fonctionnement des réseaux transnationaux de la santería cubaine (et au-delà des religions afroaméricaines) : constitués à la fois de sous-réseaux d'individus comme les ramas et de sous-réseaux de groupes comme les associations, les confréries, les « communautés » ou les familles consanguino-rituelles qui interagissent entre elles ainsi qu'avec les institutions nationales, ils offrent au regard subjectif du chercheur l'un ou l'autre de leurs aspects, induisant des interprétations parfois complètement contradictoires quant à la définition de leur mode d'organisation. La confrontation des données et la pratique d'un terrain multi-situé qui suit les « religieux » dans leurs parcours et leurs rencontres permet au contraire de mettre en évidence l'existence concrète de relations incluant différents types d'acteurs (individus, groupes, États) dont les interactions et les négociations incessantes fondent à la fois un fonctionnement en réseaux-maillage et la construction d'institutions localisées. *

* Manuscrit reçu en mars 2005, accepté pour publication en juin 2005.

\section{NOTES}

1. Culte fondé sur la dévotion et l'incorporation d'entités appelées orichas ou santos, porteurs respectivement d'un ensemble de forces ou de principes et généralement représentées sous une forme anthropomorphe possédant plusieurs facettes complémentaires, dont, entre autres, un avatar catholique. Les orichas se manifestent par la possession (appelée à Cuba " passer », " monter » ou « descendre » une entité, ou encore "se monter avec ») et par différentes techniques divinatoires, comme le lancer de morceaux de noix de coco, le lancer de cauris et les techniques liées à ifá. Outre diverses offrandes, ils ont besoin de fêtes et de sang d'animaux sacrifiés pour être contentés.

2. C'est celle qui fonde la démarche du groupe de recherche sur la transnationalisation des religions « afro-américaines » dans lequel s'inscrit ce travail. Je remercie tout particulièrement Nahayeilli Juárez Huet qui a contribuéa la réflexion concernant la partie ethnographique mexicaine dece texte. Je remercie également Stefania Capone et Élisabeth Cunin pour leurs questions stimulantes et leurs suggestions. 
3. Les Cubains disent d'un spécialiste qu'il " consulte », et d'une personne qu'elle vient " se consulter ", ou encore qu'elle vient « se fouiller », " s'occuper d'elle-même », « se voir » ou " se regarder » avec lui (consultarse, registrarse, atenderse, verse, mirarse). Ces expressions évoquent bien, de par leur construction pronominale, l'idée d'une réflexion sur soi, guidée et appuyée par une tierce personne.

4. Le spiritisme havanais sous sa forme actuelle la plus répandue vise à communiquer (par l'intermédiaire de médiums-voyants) avec des défunts au départ anonymes (parfois aussi avec des ascendants), appelés " morts de lumière ", qui « apportent de la lumière » aux vivants, et qui " prennent de la lumière » au contact de ces derniers, l'objectif étant l'« évolution » des uns et des autres.

5. Le palo et ses différentes variantes (mayombe, briyumba, kimbisa...) représentent un culte ordinairement décrit comme d'origine bantoue (entre autres), fondé sur le pacte de l'adepte avec des esprits de morts appelés nfumbe (au départ anonymes et abandonnés) et la manipulation de matériaux bruts (herbes, terres, eaux, morceaux de bois - palos -, métaux, ossements...) considérés comme porteurs de force. Les morts réclament des offrandes, des fêtes et du sang d'animaux sacrifiés pour " travailler » et se manifestent par la voyance et la possession.

6. Par ce terme (ou celui de thérapeute), j'entends désigner la personne qui consulte et propose des remèdes, quelle que soit par ailleurs la modalité de culte employée. En revanche lorsque je précise : spirite, palero ou santero, je me réfère aux personnes dont la pratique principale (la plus courante et la plus affichée) est le spiritisme, le palo ou la santería, ce qui n'exclut jamais le fait que ces personnes utilisent également les autres modalités de cultes en présence.

7. Purification ou élimination des forces néfastes. On procède par contact avec une autre source de force (d'autant plus puissante que le mal est fort) qui incorporera les forces néfastes et qui les jettera en un endroit précis.

8. À La Havane, dans le langage courant, le terme rama est également synonyme de " famille de religion » dans le sens des membres vivants contemporains. On emploie aussi avec cette dernière acception le terme de " maison " (casa ou ilé).

9. À chaque stade, le novice incorpore de nouveaux morts à travers les incisions («rayures») pratiquées en certains points de son corps, et il alimente en retour d'un peu de son sang, de sa sueur et de sa salive ses réceptacles à entités (prendas) personnelles, ainsi que le réceptacle principal (nganga) du parrain ou de la marraine. La constitution d'une nganga, fondée sur l'un des conglomérats de forces naturelles appelé " puissances » ou parfois mpungu (nommées mais non anthropomorphes, bien que certaines soient associées aux orichas principaux), est le stade ultime de l'évolution dans le palo. De cette dernière naîtront les prendas des filleul(e)s : une petite quantité de sa substance y sera incorporée.

10. Chaque entité, avec ses propres traits de caractère, attitudes, gestuelles, goûts et prohibitions, est censée correspondre à l'une des facettes de sa personnalité.

11. Terme qui désigne plus couramment les Yoruba à Cuba.

12. Dans certaines familles, on ne conçoit pas d'initiation possible sans l'action d'un parrain et d'une marraine (d'un homme et d'une femme), auxquels s'ajoute un ou une oyugbona.

13. Les spirites célèbrent avec leurs filleuls l'anniversaire du mort principal de leur "cordon spirituel » ou l'anniversaire du jour où ce dernier les a possédés pour la première fois.

14. Les foyers de la capitale ne sont pas exclusivement composés de membres liés par la consanguinité ou l'alliance matrimoniale. Ils s'organisent en général autour d'un personnage central. Les conjoints peuvent changer, les enfants partir ou revenir, amener avec eux d'autres enfants, accueillir un ami sans toit, un parent éloigné, un filleul de religion et ses proches : toutes les combinaisons sont imaginables, pourvu que l'accord tacite du (ou de la) chef de famille ait été obtenu.

15. Les échanges de biens sont toutefois peu réalisés dans ce cadre.

16. Mèche de cheveux du novice conservée après le rasage du sommet du crâne, ciseaux ayant servi à la couper, plat dans lequel le novice a mangé... Ces assiettes servent parfois d'ornement dans la maison du parrain ou de la marraine, leur nombre étant source d'orgueil et de prestige.

17. Incluant l'idée d'un échange bénéfique pour toutes les parties : «Quand tu es en train de servir les autres dans une activité de religión, tu évolues, tes morts évoluent, autrement dit ça t'aide, toi » explique une femme spirite et palera. 
18. Même si les femmes ménopausées ayant passé le grade initiatique correspondant peuvent théoriquement sacrifier des animaux, la plupart du temps ce sont les hommes qui effectuent cette opération. Actuellement, à ma connaissance, il n'existe pas, non plus, de femme obbá à La Havane.

19. En octobre 1991, lors du IV ${ }^{e}$ Congrès du Parti communiste cubain, suite à la crise provoquée par la chute de l'URSS et l'arrêt des échanges économiques privilégiés avec les pays de l'Est, l'accès au Parti des religieux de toutes obédiences a été autorisé (Argyriadis 1999, pp. 274-288).

20. L'épicerie (bodega) est un établissement proche du domicile auquel est attaché le carnet de rationnement d'un foyer et où sont distribuées aux premiers arrivés des denrées supplémentaires, à prix d'État. Les produits vendus dans les marchés libres paysans sont très chers, fluctuent parfois d'un quartier à l'autre et ne sont pas approvisionnés de la même façon. On peut donc aussi échanger des renseignements à leur sujet, de même pour les médicaments disponibles ou non d'une pharmacie à une autre.

21. Cette réflexion découle précisément d'observations faites à Paris, Barcelone et Mexico. Le cas de villes comme Miami est sans doute très différent.

22. En 1993, c'est-à-dire au plus fort de la crise qu'a connue le pays, lorsque le Centre d'anthropologie de l'Académie des sciences de Cuba a mené une enquête sur les "relations raciales » dans le quartier Cerro, le questionnaire comportait la question suivante : " Votre (ou vos) meilleur(s) ami(es) sont-ils Blancs, Noirs ou Mulâtres ? ». À $99 \%$, les personnes interrogées ont répondu qu'elles n'avaient pas d'amis : "Amitié, amitié, cet ami-là dont on peut dire qu'il est un frère pour toi ? Non, rien ».

23. Pour que le tableau soit complet, il faudrait également aborder la question des sociétés secrètes masculines, comme la société secrète abakuá (López Valdés 1985) ou les loges maçonniques.

24. Les travaux récents de Barcia Zequeira (2003) montrent toute l'importance du rôle joué par les liens de parenté "par affinité » (cabildo, parrainage, tutorat...) dans l'organisation familiale des esclaves et de leurs descendants au XIX ${ }^{\mathrm{e}}$ siècle.

25. À quelques variantes près, les leaders se réclament tous des lignages rituels des mêmes " grands " ancêtres, outil de légitimation d'autant plus essentiel qu'il intègre toujours des personnages rendus célèbres par la littérature orale et ethnographique.

26. Cette capacité repose d'ailleurs plus sur des fondements idéologiques virtuels que sur des rencontres régulières et des échanges concrets. En dehors des moments de collaboration médiatisés (cérémonie de la "Lettre de l'année ", colloques, déclarations communes à la presse au gré des événements...), les membres d'une même association se voient peu et sont mis au courant des activités des uns et des autres par des voies de communication communes à tous : réseau de socios et réseau de ramas.

27. Pour l'instant, les données manquent sur l'origine sociale des santeros mexicains. La question de l'influence de cet élément sur le fonctionnement interne des familles de religion mexicaines reste à creuser. Dans l'un des cas étudiés, à Veracruz, j'ai pu observer la façon dont une marraine de palo et de santo met en contact ses clients de consultation et ses filleuls en fonction des problèmes à résoudre (un avocat avec un commercial, un médecin avec un élu local, etc.). Mais à ce stade de l'enquête rien ne permet d'indiquer si la hiérarchie rituelle prime sur la hiérarchie sociale ou si elle la renforce.

28. Les sacrifices d'animaux posent problème, mais aussi le manque d'ingrédients et de correligionaires. En outre, même dans le cas des villes où les santeros cubains sont nombreux et où la pratique est courante, comme Mexico ou Miami, un rituel effectué à Cuba aura toujours une plus grande valeur aux yeux de leurs concitoyens (et il coûtera moins cher). Les films vidéos sont alors utilisés comme preuve validant la cérémonie.

29. Ce qui, dans le cas des Mexicains, est loin d'être toujours vrai.

30. D'autres se raccordent au candomblé brésilien et « transforment leurs saints lucumí en orixás de candomblé » (Juárez Huet 2004, p. 73).

31. Qui, en effet, au Mexique, est dans un premier temps le seul à détenir ces privilèges et peut se targuer d'occuper une position hiérarchique supérieure à celle qu'il occupe réellement dans le réseau " religieux ». Plusieurs tactiques sont possibles : jouer sur l'âge d'initiation (le terme iniciación est ambigu ; il désigne au Mexique une première série d'acquisition d'objets rituels alors qu'à Cuba il fait 
référence à la fixation de l'oricha dans la tête), affirmer avoir pour parrain ou marraine une personne de grande renommée quand, en réalité, ladite personne était simplement présente lors de l'initiation, prétendre avoir de nombreux filleuls... À La Havane, chacun a tôt fait de vérifier les allégations des uns et des autres par le biais des discussions qui occupent un large temps, pour ne pas dire l'essentiel des cérémonies, où circulent les religieux de plusieurs familles. Au Mexique, cela n'est plus possible et chacun, quelle que soit sa nationalité, joue avec l'ouverture que permet le contexte d'éloignement.

\section{RÉFÉRENCES BIBLIOGRAPHIQUES}

Amselle Jean-Loup

2001 Branchements. Anthropologie de l'universalité des cultures, Flammarion, Paris.

ANDRÉ Jacques

1987 L'inceste focal dans la famille noire antillaise, PUF, Paris.

Appadurai Arjun

1996 Modernity at large. Cultural dimension of globalization, University of Minnesota Press, Minneapolis-Londres.

\section{ArgYriadis Kali}

1999 La religión à La Havane. Actualité des représentations et des pratiques cultuelles havanaises, Éditions des Archives contemporaines, Paris.

2001-2002 «Les Parisiens et la santería : de l'attraction esthétique à l'implication religieuse », Psychopathologie africaine, XXXI (1), pp. 17-44.

2006 «Les batá deux fois sacrés. La construction de la tradition musicale et chorégraphique afro-cubaine ", Civilisations, LIII (1-2), pp. 45-74.

\section{Argyriadis Kali et Stefania CAPONE}

2004 «Cubanía et santería. Les enjeux politiques de la transnationalisation religieuse (La Havane-Miami) ", Civilisations, LI (1-2), janvier, pp. 81-137.

Barcia Zequeira María del Carmen

2003 La otra familia. Parientes, redes y descendencia de los esclavos en Cuba, Casa de las Américas, La Havane.

BASTIDE Roger

1967 Les Amériques noires, Payot, Paris.

Benoist Jean

1984 "L'organisation sociale des Antilles », in L'Afrique en Amérique latine, UNESCO, Paris, pp. 61-81.

CAPONE Stefania

2004 "À propos des notions de globalisation et de transnationalisation », Civilisations, LI (1-2), janvier, pp. 9-22.

2005 Les Yoruba du Nouveau Monde. Religion, ethnicité et nationalisme noir aux États-Unis, Karthala, Paris. 
CAPONE Stefania, éd.

2001-2002 Les pratiques européennes des religions afro-américaines, numéro spécial de Psychopathologie africaine, XXXI (1).

2004 Religions transnationales, numéro spécial de Civilisations, LI (1-2).

Colonomos Ariel

1995 Sociologie des réseaux transnationaux. Communautés, entreprises et individus : lien social et système international, L'Harmattan, Paris.

Degenne Alain et Michel Forsé

1994 Les réseaux sociaux. Une analyse structurale en sociologie, Armand Colin, Paris.

DE LA TORRE Renée et José Manuel Mora

2001 "Itinerarios creyentes del consumo neoesotérico », Comunicación y Sociedad, 39, enero-junio, pp. 113-143, DECS, Universidad de Guadalajara.

FIRTH Raymond

1954 "Social organization and social change », Journal of the Royal Anthropological Institute, LXXXIV, pp. 1-20.

Forsé Michel

1991 "Les réseaux de sociabilité : un état des lieux ", L'Année Sociologique, 41, pp. 247-264.

Foster George M.

1963 "The dyadic contract », American Anthropologist, 63, pp. 1173-1192.

GoBin Emma

2003 Les religions d'origine africaine à La Havane. Ethnologie d'une harmonie rêvée, mémoire de maîtrise, université de Paris X, Nanterre.

Granovetter Mark

2000 "La force des liens faibles », in Mark Granovetter, éd., Le marché autrement, Desclée de Brouwer, Paris, pp. $45-73$ [1973].

Gueds Pauline

2004 " "A Nation within Nations" : nationalisme afro-américain et réafricanisation aux États-Unis », Civilisations, LI (1-2), janvier, pp. 23-38.

JuÁrez HuET Nahayeilli

2004 «La santería dans la ville de Mexico : ébauche ethnographique », Civilisations, LI (1-2), janvier, pp. 61-79.

LACHATEÑERÉ Romulo

2001 «Las creencias religiosas de los afrocubanos y la falsa aplicación del término brujería ", in Romulo Lachateñeré, éd., El sistema religioso de los afrocubanos, Ed. Ciencias Sociales, La Havane, pp. 196-216 [1939].

López VALDÉs Rafael L.

1985 "La sociedad abakuá en un grupo de trabajadores portuarios », in Rafael L. López Valdés, éd., Componentes africanos en el etnos cubano, Ed. Ciencias Sociales, La Havane, pp. 151-185. 


\section{MENÉNDEZ Lázara}

2002 Rodar el coco. Proceso de cambio en la santería, Ed. Ciencias Sociales, La Havane.

\section{ORTIZ Fernando}

1939 « Brujos o santeros », Estudios Afrocubanos, III (1-4), pp. 85-90, La Havane.

1992 Los Cabildos y la fiesta afrocubana del dia de Reyes, Ed. Ciencias Sociales, La Havane [1920].

TESTA Silvina

2005 "La "lucumisation" des cultes d'origine africaine à Cuba : le cas de Sagua la Grande ", Journal de la Société des Américanistes, 91-1, pp. 113-138. 


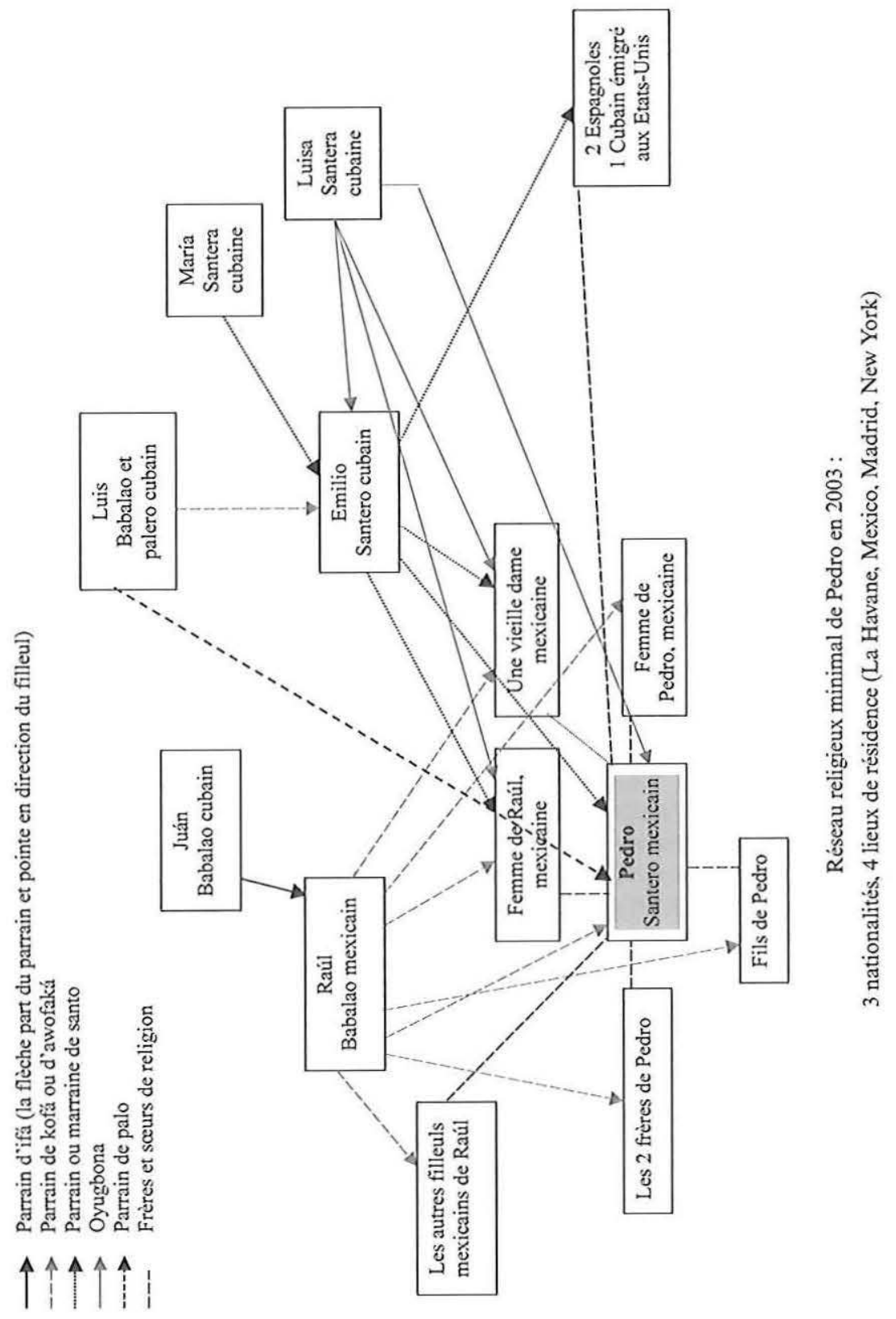




\section{NOTE ETHNOGRAPHIQUE EXPLICATIVE}

Pedro a eu 34 ans en août 2003, il est Mexicain et vit à Mexico avec sa femme et son fils. Il travaille au noir comme intermédiaire entre des agents des douanes de la frontière nord et les acheteurs éventuels de marchandises confisquées. Il raconte comment, trois ans auparavant, un client libanais lui a parlé de son parrain de santo, un babalao cubain qui vivait comme lui à Mexico. Ensemble ils lui ont rendu visite, mais Pedro a trouvé le prix de la consultation exagéré : environ 300 dollars. Il avoue cependant avoir été très impressionné par les autels et les réceptacles à orichas et intéressé par les possibilités de développer son commerce et gagner de l'argent grâce aux pouvoirs de ces derniers. Un peu plus tard, un autre client, mexicain, l'a emmené chez un palero cubain qui lui a passé un coq vivant sur le corps, puis lui a ordonné de s'enfuir dans la rue, ce qui a effrayé Pedro. Enfin il a fait la connaissance de Raúl, un Mexicain babalao initié à Cuba, qui lui a donné, ainsi qu'à son fils, ses deux frères et son épouse, l'initiation mineure en ifá (awofaká ou kofá). Il s'en dit satisfait, suite à la mort d'un concurrent de ses frères, qu'il soupçonnait de les avoir ensorcelés.

Avec Raúl, Pedro est allé six fois à La Havane. La première fois il s'est initié au palo avec Luis, que Jorge a connu par l'intermédiaire de son propre parrain d'ifá. Luis souhaitait que Pedro s'initie à la santería avec l'une de ses filles, mais Pedro a fini par porter son choix sur l'un des filleuls d'awofaká de Luis, Emilio, qu'il avait rencontré pendant une cérémonie et dont il appréciait le sérieux. Emilio s'occupait en effet d'une association visant à compiler les textes écrits par les religieux pour transmettre une partie de leurs connaissances à leurs filleuls. Il était en rapport avec plusieurs chercheurs cubains, américains et européens, avait fait une conférence au Canada et avait déjà parrainé deux femmes espagnoles.

Pedro s'est donc initié à la santería avec Emilio et avec l'ayugbona de celui-ci comme ayugbona. Par la suite, Emilio a également initié la femme de Raúl, une de ses filleules d'awofaká et enfin un Cubain qui a émigré juste après à New York. Au cours de ces rituels, Pedro a fait la connaissance d'un autre babalao cubain, Francisco, qui travaillait avec Emilio et qui est devenu l'interlocuteur principal de Raúl en ce qui concerne ifá, au détriment de son parrain d'origine. Des tensions ont surgi entre Pedro et Emilio, notamment parce que Pedro pensait qu'Emilio ne voulait pas le laisser devenir babalao. Emilio, fort de son savoir livresque et de sa nationalité, était également en constant désaccord avec Raúl sur la façon dont il convenait de mener les cérémonies, malgré le fait que ce dernier soit babalao. Pedro trouve que ce n'est pas bien, car « légalement » il doit respecter la hiérarchie. Il émet aussi des doutes sur la moralité de la famille d'Emilio. Au bout d'un an qu'il considère comme catastrophique (du point de vue professionnel), il est retourné à La Havane sans en informer Emilio et « s'est consulté » avec Francisco, qui lui a fait comprendre qu'Emilio l'attaquait en sorcellerie. Il lui a par ailleurs proposé de l'initier à ifá.

Pedro a définitivement rompu avec Emilio et commencé à réunir l'argent et les ingrédients nécessaires pour sa nouvelle initiation, tout en maintenant son lien à Raúl, indispensable pour l'aider à mener des rituels à Mexico. Il envisage d'initier par la suite lui-même ses frères, et de fonder une famille de religion constituée exclusivement de proches. Il se documente sur la santería grâce aux livres qu'il a achetés à Cuba, et commence à explorer les sites internet et à s'intéresser au tradicionalismo (prônant l'hégémonie rituelle des Yoruba nigérians). Malgré leur brouille, Emilio et Pedro sont très au courant de leurs 
activités respectives (via leurs multiples parents rituels communs), interprétant chaque infortune de l'autre comme une conséquence du manque de respect des relations qui sont censées prévaloir entre parrain et filleul.

L'exemple du réseau religieux minimal de Pedro montre toute la complexité et la mobilité des liens (positifs et négatifs) qui unissent entre eux les membres d'une même rama, et ce malgré leur éloignement géographique et leurs différentes nationalités. Pour que le tableau soit complet, il faudrait inclure au moins tous les frères et souurs de religion dans chacune des modalités de culte, mais l'espace imparti ici ne permet pas un tel développement. Précisons également que le cas de Pedro a été choisi pour sa simplicité, liée à son histoire religieuse courte (trois ans). 